Research Paper

\title{
Overall survival and post-progression survival are potent endpoint in phase III trials of second/third-line chemotherapy for advanced or recurrent epithelial ovarian cancer
}

\author{
Mototsugu Shimokawa ${ }^{\bowtie}$, Takahiro Kogawa ${ }^{2 \bowtie}$, Takako Shimada ${ }^{3}$, Toshiaki Saito ${ }^{4}$, Hozumi Kumagai ${ }^{5}$, \\ Masafumi Ohki ${ }^{6}$, Tsunehisa Kaku ${ }^{6}$
}

1. Cancer Biostatistics Laboratory, Clinical Research Institute, National Kyushu Cancer Center, Fukuoka, Japan;

2. Department of Developmental Therapeutics, National Cancer Center Hospital East, Chiba, Japan;

3. Department of Obstetrics and Gynecology, Nagasaki University Graduate School of Biomedical Sciences, Nagasaki, Japan;

4. Gynecologic Service, National Kyushu Cancer Center, Fukuoka, Japan;

5. Department of Medical Oncology, National Kyushu Medical Center, Fukuoka, Japan;

6. Department of Health Sciences, Graduate School of Medical Sciences, Kyushu University, Fukuoka, Japan.

$\triangle$ Corresponding author: Dr. Mototsugu Shimokawa, Cancer Biostatistics Laboratory, Clinical Research Institute, National Kyushu Cancer Center, 3-1-1 Notame, Minami-ku, Fukuoka 811-1395, Japan. Tel: +81-92-541-3231; Fax: +81-92-541-8534; E-mail: shimokawa.m@nk-cc.go.jp and Dr. Takahiro Kogawa, Department of Developmental Therapeutics, National Cancer Center Hospital East, 6-5-1 Kashiwanoha, Kashiwa, Chiba 277-8577, Japan. Tel: +81-4-7133-1111; FAX: +81-4-7131-5390; E-mail: tkogawa@east.ncc.go.jp

(C) Ivyspring International Publisher. This is an open access article distributed under the terms of the Creative Commons Attribution (CC BY-NC) license (https://creativecommons.org/licenses/by-nc/4.0/). See http://ivyspring.com/terms for full terms and conditions.

Received: 2016.09.21; Accepted: 2018.01.18; Published: 2018.02.16

\begin{abstract}
Purpose: A growing number of treatment options and active compounds in treatments have led to better outcomes for patients with advanced or recurrent epithelial ovarian cancer. We examined the association between progression-free survival (PFS), post-progression survival (PPS) and overall survival (OS) in phase III trials of second- and third-line chemotherapy for advanced or recurrent epithelial ovarian cancer. We aim to determine whether PFS or PPS is a surrogate of OS so that we can decide progress of disease is optimal endpoint for ovarian cancer.

Methods: We identified 22 trials conducted between January 1, 2000 through December 31, 2014 by literature search. We divided OS into PFS and PPS, and assessed the association between OS and PFS/PPS. We also examined whether the year of trial enrollment completion was associated with any variables.

Results: The median PPS was slightly longer in recent trials compared to older trials (10.0 vs. 8.8 months). While PPS was strongly associated with OS $(r=0.88)$ in all trials, PFS was moderately correlated with OS $(r=0.72)$. The correlation between OS and PPS in recent trials $(r=0.93)$ was stronger than in older trials $(r=0.84)$.

Conclusions: Our findings indicate that PPS is highly associated with OS in second/third-line chemotherapy for advanced or recurrent epithelial ovarian cancer, while the association between PFS and OS is moderate. We recommend using OS as primary endpoint for clinical trial of ovarian cancer, however PFS is still an optional endpoint.
\end{abstract}

Key words: Ovarian Cancer, Chemotherapy, Overall Survival, Post-Progression Survival, Phase III trial

\section{Introduction}

Ovarian cancer is regarded as one of the most lethal neoplasm of all the gynecologic malignancies [1]. According to the American Cancer Society, an estimated 21,290 new cases of ovarian cancer and 14,180 deaths from the disease are expected in the U.S. in 2015 [2]. Epithelial ovarian cancer accounts for 
about $90 \%$ of all ovarian cancers. Most epithelial ovarian cancers are not diagnosed until they are at an advanced stage, resulting in a poor prognosis [3]. However, among all gynecologic cancers, this cancer has the highest response rate to chemotherapy. Many patients who undergo a combination of surgery and chemotherapy achieve complete remissions [4].

Although epithelial ovarian cancer responds well to first-line chemotherapy, recurrence occurs for more than half of these patients within two years after the chemotherapy. For advanced cancers (e.g. stage III/IV), 55\% recur within two years, and over $70 \%$ of patients does within five years [5]. Median overall survival (OS) after recurrence is approximately two years. Post-recurrence treatment goals differ from that of first-line treatments owing to the difficulty for achieving a complete remission. These goals aim at extending OS as well as improving quality of life (QOL) and alleviating symptoms [6]. Chemotherapy is the main treatment option available for advanced or recurrent ovarian cancers. However, it remains unclear which chemotherapeutic agents contribute the most to a favorable prognosis.

Phase III clinical trials in oncology have several purposes, and these are (i) to compare new treatments to standard therapy, (ii) to determine how they affect patient QOL, (iii) to determine toxicity profiles, and (iv) to evaluate the economic consequences of implementing the new treatments. OS has long been regarded as reliable endpoint when selecting an optimal treatment regimen for cancer patients, however the increase in the number of available treatment options effected on OS time. A number of clinical trials for second- or third-line treatments of ovarian cancer, have been utilizing progression-free survival (PFS) as a primary endpoint instead of OS. However, significant improvement in PFS may not translate into a similar improvement in OS, it would be inappropriate to conclude that a non-statistically significant OS outcome in a randomized trial is evidence for the lack of clinical utility of an investigative approach that has been determined to improve PFS. [7]. Therefore, it is critical to understand the impact of therapeutic modalities after disease has progressed. Emerging evidence demonstrated the influence of post-progression therapies on patient outcomes, and PPS was expected to perform legitimate determinant of OS. In a study of phase III trials of first-line chemotherapy for advanced epithelial ovarian cancer, PPS was found to be more highly associated with OS than PFS, especially in recent trials [8]. Despite the favorable response to first-line chemotherapy in ovarian cancer, disease progression is frequent, and many receive second- and third-line treatments. However, little is known about the actual implications of PPS in second/thirdline chemotherapy for this disease. We hypothesized OS is more appropriate endpoint compared with PFS among ovarian epithelial carcinoma treated with second/third-line chemotherapy.

For this study, we separated OS from phase III randomized controlled trials for second/third-line treatment of patients with advanced or recurrent epithelial ovarian cancer into PFS and PPS, and assessed the association of each variable with OS.

\section{Materials and Methods}

\section{Literature search}

We performed literary search, and selection process was illustrated in CONSORT diagram (Figure 1). We conducted a PubMed search (US National Library of Medicine) between January 1, 2000 through December 31, 2014. Search keywords included "ovarian carcinoma," "ovarian cancer," "clinical trial," and "chemotherapy." We only chose phase III randomized controlled trials and articles published in English. Selected publications for review compared at least two second- or third-line systemic chemotherapies. Treatments involving molecular-targeted agents for advanced epithelial ovarian cancer were included. In addition, we searched the reference lists of included trials and large, systematic reviews. Regardless of the availability of explicitly defined parameters, trials providing data for both OS and either PFS or time to progression (TTP) were included. Trials investigating immunotherapy regimens or hormonal therapies were excluded to exclude possible bias.

\section{Data abstraction and clinical endpoint}

Primary endpoints were analyzed in detail according to the definitions provided by the studies' authors. The primary endpoint used for sample size calculation was used when no specific statement was provided by the authors. In line with recent reporting trends, we collectively referred the two endpoints (PFS and TTP) based on tumor assessment simply to PFS $[9,10]$. Median OS and median PFS were extracted from all trials when data were available. The definition for median PPS was median OS minus median PFS for each trial. We obtained the following information from each report: i) the year of trial enrollment completion, ii) the line of treatment, iii) the number of patients in each treatment arm, iv) the number of treatment arms in each trial, v) the types of agents, vi) the ages of patients and vii) the number of patients with clear cell carcinoma. 


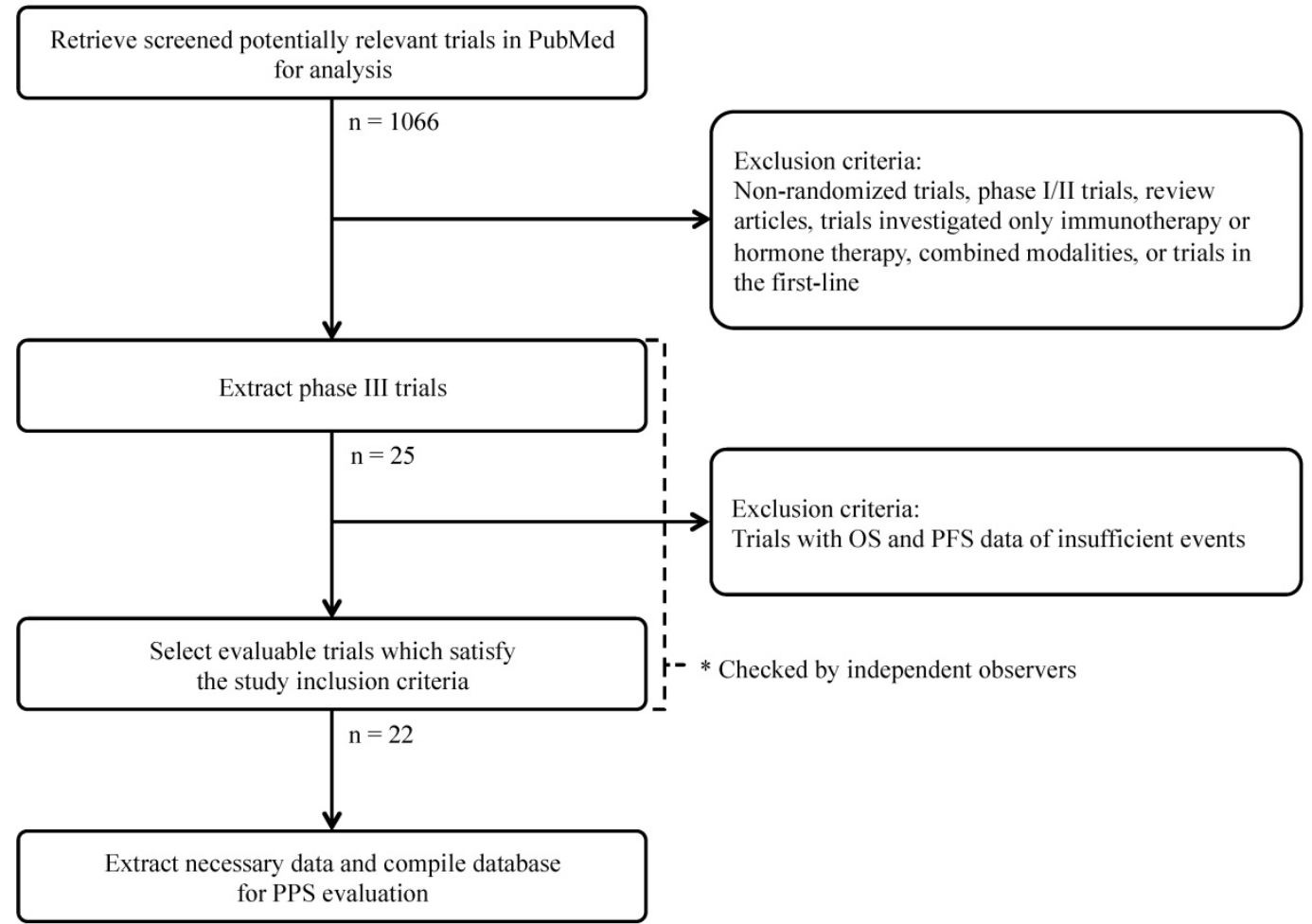

Figure 1. CONSORT diagram of the search strategy and selection of phase III randomized controlled clinical trials for advanced or recurrent epithelial ovarian cancer.

The aims of this analysis were: to evaluate the correlation of OS and (PFS/PPS) in phase III randomized controlled trials of second/third-line chemotherapy for advanced or recurrent epithelial ovarian cancer, and to determine whether the aforementioned correlations changed depending on when trial enrollment was completed (before or after May 2003).

\section{Statistical analysis}

For all trial arms, the median was used to summarize the survival data. For each trial arm, the proportion of OS accounted for by PPS was calculated as: 100 (1 - median PFS [mPFS]/median OS [mOS]). Spearman's rank correlation coefficient was used to assess the association between mOS and either mPFS or median PPS (mPPS). Analyses were weighted by the number of patients in each arm to account for differences in sample size and patient characteristics among trial arms.

Additionally, all trials were divided into two groups on the basis of when trial enrollment was completed. Given that the median date of enrollment completion for the 22 analyzed trials [11-32] was April 16, 2003, trials were divided around this date (older trials included those from February 1995 until March 2003, and recent trials included those from April 2003 to April 2011) in order to evaluate a possible change in PPS, and to assess whether trial results were dependent upon the year of trial enrollment completion. Differences in the survival data between older and recent trials were determined by comparing the medians of survival data with the Wilcoxon rank-sum test. All reported $p$-values correspond to two-sided tests. A $p$-value of $<0.05$ was considered statistically significant. Analyses were performed in SAS 9.4 (SAS Institute, Cary, NC).

\section{Results}

\section{Characteristics of the trials}

The literary search identified a total of 1,066 potentially relevant publications. Of these, 1,044 studies were excluded based on one or more of the following reasons: investigation of only immunotherapy regimens or hormonal therapies; examination of other malignancies or combined modality treatments (e.g. radiotherapy); non-randomized study; phase I or II trials; review articles, letters or commentaries; portrayal of subgroup analyses derived from other trials or equivalent content to similar retrieved studies; and phase III randomized controlled trials investigating first-line chemotherapy treatments. Review of the remaining publications yielded 22 trials, which satisfied the inclusion criteria for this study.

The main characteristics of these 22 phase III randomized, controlled clinical trials are summarized 
(Table 1). Treatment regimen assessed in this study is described in Supplementary table 1. A total of 8,121 patients with advanced or recurrent epithelial ovarian cancer were enrolled, with a median number of 313 (range 61-976) patients per study. The average age of patients was 59.8 years. A total of 13 trials used a primary endpoint based on tumor assessment (PFS or TTP), whereas eight trials used OS as the primary endpoint. In one trial, both OS and PFS were primary endpoints. Clear cell carcinoma is generally assumed as chemotherapy resistant tumor compared to serous adenocarcinoma, and we confirmed the proportion of this disease was low (Supplementary Table 1). Advanced or recurrent epithelial ovarian cancer patients can be categorized as either platinumsensitive or platinum-resistant with regard to their response to platinum-based chemotherapy agents. Trials containing either platinum-sensitive $(n=9)$ or platinum-resistant $(\mathrm{n}=8)$ patients, as well as both $(n=5)$, were included.

Table 1. Characteristics of the selected 22 phase III randomized controlled clinical trials for advanced or recurrent epithelial ovarian cancer

\begin{tabular}{ll}
\hline Trial characteristics & \\
\hline Median number of patients per trial & $313(61-976)$ \\
$\begin{array}{l}\text { Average of median age (years) } \\
\text { Median number of serous adenocarcinomab }\end{array}$ & 59.8 \\
$\begin{array}{l}\text { Median number of clear cell carcinomac } \\
\text { Primary endpoint (number of trials) }\end{array}$ & $101(25-366)$ \\
OS & $5.5(0-28)$ \\
PFS or TTP & 8 \\
Other & 13 \\
Classify types of platinum sensitivity (number of trials) & 1 \\
Sensitive to platinum & 9 \\
Resistant to platinum & 8 \\
Both sensitive and resistant to platinum & 5 \\
\hline $\begin{array}{l}\text { aFour trials were excluded (data not shown) } \\
\text { bTen trials were excluded (data not shown) } \\
\text { c14 trials were excluded (data not shown) }\end{array}$
\end{tabular}

\section{Median OS, PFS, and PPS of all trials and subgroups based on year of trial enrollment completion (older trials, up to April 2003; recent trials, April 2003 and later)}

The survival data of all trials and trial arms according to the year of trial enrollment completion are summarized in Table 2. Trial enrollment completion dates were extracted from the study publications or their preceding publications when available. One study [17] had no trial enrollment completion data and was assigned January 1 of the year of the initial study publication [33]. Similarly, a study [25] that only provided the year of trial enrollment completion was assigned January 1 of the given year.
Table 2. Survival data for all trials and trial arms grouped according to the year of trial enrollment completion

\begin{tabular}{|c|c|c|c|c|}
\hline Item & $\begin{array}{l}\text { Overall } \\
\mathrm{n}=44\end{array}$ & $\begin{array}{l}\text { 2nd/3rd-line } \\
\text { (up to April2003) } \\
n=21\end{array}$ & $\begin{array}{l}\text { 2nd/3rd-line } \\
\text { (April2003 } \\
\text { and later) } \\
\mathrm{n}=23\end{array}$ & $p$-value* \\
\hline \multicolumn{5}{|l|}{ PFS(months) } \\
\hline Median (Range) & $5.8(2.3-22.0)$ & $6.0(3.2-22.0)$ & $\begin{array}{l}5.0(2.3-12.0 \\
\end{array}$ & 0.0540 \\
\hline \multicolumn{5}{|l|}{ PPS(months) } \\
\hline Median (Range) & $9.7(3.6-34.0)$ & $8.8(3.6-34.0)$ & $\begin{array}{l}10.0(5.7- \\
28.7)\end{array}$ & 0.3533 \\
\hline \multicolumn{5}{|c|}{ OS(months) } \\
\hline Median (Range) & $14.6(8.5-53.0)$ & $14.2(10.3-53.0)$ & $\begin{array}{l}15.2(8.5- \\
33.2)\end{array}$ & 0.7600 \\
\hline \multicolumn{5}{|c|}{ PPS/OS (\%) } \\
\hline Median (Range) & $63.3(25.6-86.7)$ & $58.5(25.6-72.2)$ & $\begin{array}{l}68.1(55.6 \text { - } \\
86.7)\end{array}$ & 0.0050 \\
\hline
\end{tabular}

Across all arms $(n=44)$, the mOS was 14.6 months, while the mPFS and mPPS were 5.8 and 9.7 months, respectively. Across older trials $(n=21)$, the mOS, mPFS and mPPS were 14.2, 6.0 and 8.8 months, respectively. Across recent trials $(\mathrm{n}=23)$, the mOS, mPFS and mPPS were 15.2, 5.0 and 10.0 months, respectively. The mPFS was slightly shorter in recent trials compared to older trials (5.0 and 6.0 months, respectively, $p=0.0540$ ), and the average MPPS was not significantly different between recent trials and older trials (10.0 and 8.8 months, respectively, $p=$ 0.3533). The proportion of mOS accounted for by mPPS significantly increased from $58.5 \%$ in older trials to $68.1 \%$ in recent ones $(p=0.0050)$.

In evaluation of survival time according to platinum sensitivity, there were small number of study arm in either platinum-resistant $(n=16)$ or platinum-sensitive $(n=17)$ category and it was difficult to perform separate analyses according to platinum sensitivity, however the ratio of PPS/OS showed similar trend between platinum-resistant $(64.9 \%)$ and platinum-sensitive (61.3\%) (Supplementary Table 2).

\section{Relation between OS and either PFS or PPS}

Figures 2 and 3 show the association between mOS and either mPFS or mPPS for all trials (44 arms, PPS/OS ratio: $63.3 \%$ ), respectively. The mPPS was strongly correlated with median OS $(r=0.88, p<$ $0.0001)$ as indicated by Spearman's correlation coefficient, whereas mPFS was moderately correlated with median OS $(r=0.72, p<0.0001)$.

The correlation between median OS and median PFS in recent trials $(r=0.64, p<0.0001)$ has weakened compared to older trials $(r=0.81, p<0.0001)$ and the correlation between mOS and MPPS in recent trials $(r$ $=0.93, p<0.0001)$ is stronger in comparison to older trials $(r=0.84, p<0.0001)$. 
A

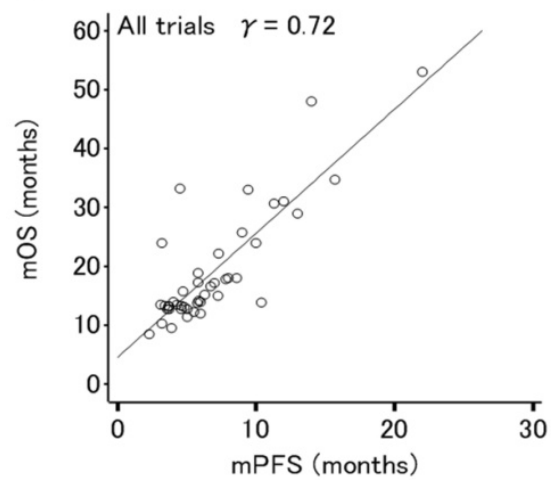

B

C
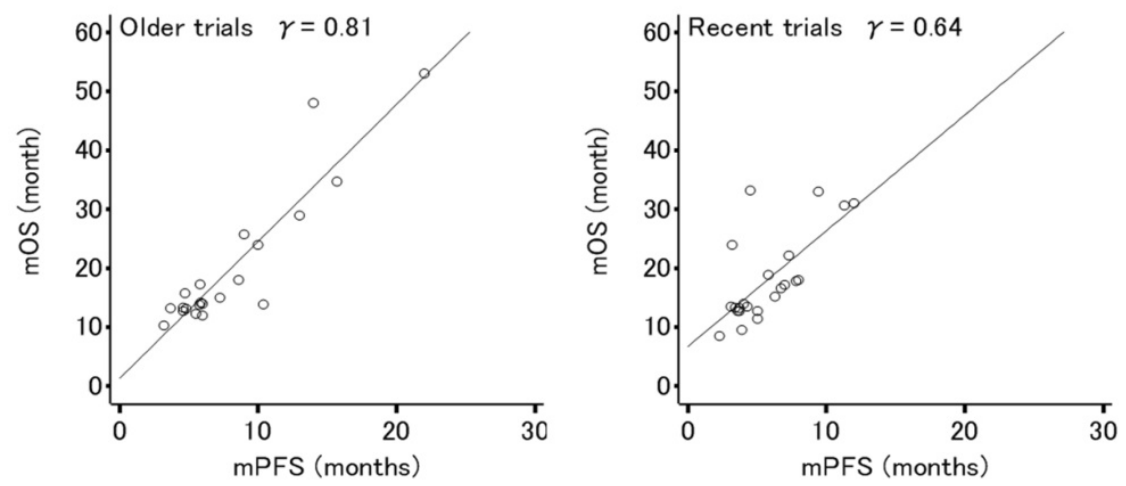

Figure 2. Correlation analyses for the relation between median overall survival (OS) and median progression-free survival (PFS) in 44 arms evaluating patients $(n=8,121)$ of 22 phase III trials for advanced or recurrent epithelial ovarian cancer. (A) All trials. (B) Older trials (trial enrollment completed between FEB1995 and APR2003). (C) Recent trials (trial enrollment completed between APR2003 and APR2011). Each dot area is proportional to the number of patients in each trial arm. The $r$ values displayed are Spearman's rank correlation coefficient.

A

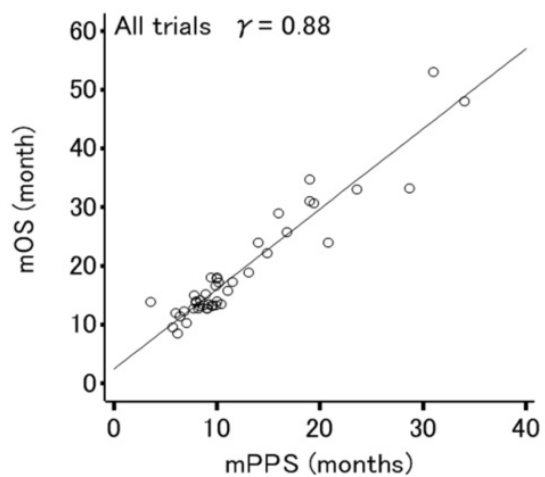

B

C
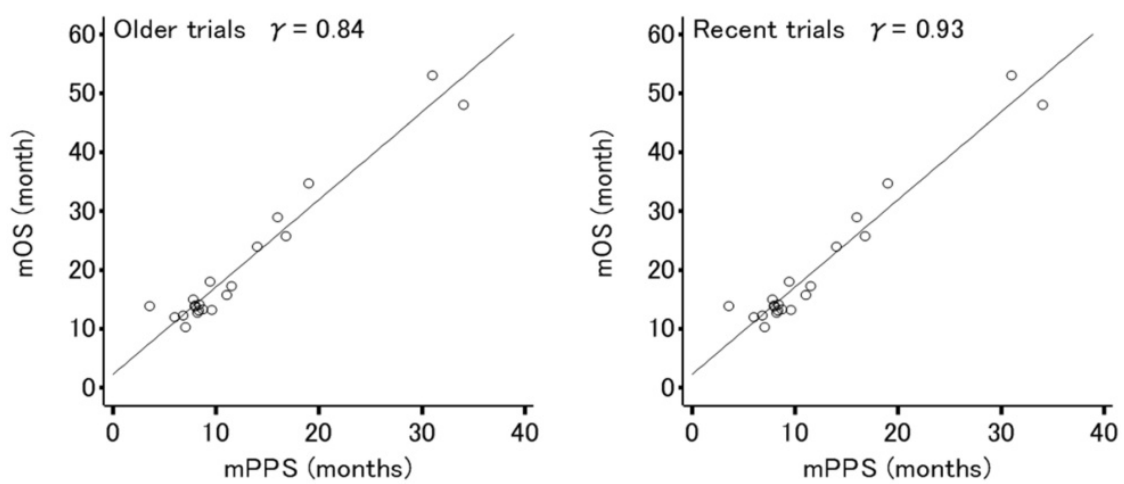

Figure 3. Correlation analyses for the relation between median overall survival (OS) and median post-progression survival (PPS) in 44 arms evaluating patients $(n=8,121)$ of 22 phase III trials for advanced or recurrent epithelial ovarian cancer. (A) All trials. (B) Older trials (trial enrollment completed between FEB1995 and APR2003). (C) Recent trials (trial enrollment completed between APR2003 and APR2011). Each dot area is proportional to the number of patients in each trial arm. The $r$ values displayed are Spearman's rank correlation coefficient. 


\section{Discussion}

OS has long been regarded as the gold-standard primary endpoint in assessing cancer treatment efficacy. However, a significant number of phase III clinical trials assessing first-line treatment for ovarian cancer have used PFS as a primary endpoint. While PPS has been found to be more highly associated with OS than PFS, especially in recent trials of first-line treatment for advanced or recurrent ovarian cancer [8], it remains unclear whether this holds true in the second- or third-line settings. Concerns about the use of PFS as a surrogate endpoint for OS are that PFS is highly tumor-dependent as well as treatmentdependent, in which surrogacy may need to be re-established for every new treatment class. Oza et al. have proposed three situations in advanced ovarian cancer where PFS is the preferred primary endpoint: i) when crossover or contamination of OS is expected, ii) when the absolute gain in PFS is clinically relevant, and iii) when PFS is supported by one or more additional endpoints such as OS or QOL [34].

Although PFS contains information about OS, PPS plays a critical role for making inferences about OS [35]. For instance, PPS has been reported to be influenced by progression patterns and to serve as a key in prognostic prediction as well as design of second-line trials in hepatocellular carcinoma [36]. In this study, the mPPS constituted more than half of the mOS, and the median proportion of OS accounted for by PPS increased by $9.6 \%$ over time $(p=0.0050)$. Our result demonstrated that $\mathrm{MPPS}$ is strongly associated with mOS, while mPFS show modest correlation with mOS especially in recent trials.

Other first-line trials have investigated the association between OS and PFS or PPS. In gastric and colorectal cancer, PFS has been approved as a surrogate endpoint on the basis that both diseases see a prolonged OS in patients as well as evidence of a strong correlation between OS and PFS $[37,38]$. In advanced colorectal cancer, PFS was suggested as an appropriate surrogate for OS when improvements in OS accompanied improvements in PFS [39]. However, results from phase III clinical trials of first-line treatments for advanced NSCLC [10], along with breast [9], colorectal [39], and ovarian [34] cancer are similar to ours and describe a modest association between PFS and OS compared to a strong association between PPS and OS.

To the best of our knowledge, this is the first study to investigate the association between OS and PFS or PPS in phase III randomized controlled clinical trials of second/third-line treatments for advanced or recurrent ovarian cancer. No definitive correlation between OS and PFS has been established in second- or third-line ovarian cancer treatment. When we assessed PFS and PPS, we can simultaneously demonstrate the association with OS. We encourage them to use OS as a primary endpoint rather than PFS because the association was strong, however PFS showed modest association with OS and salvage treatment may confound PPS. We should note PFS is still an optional surrogate marker for OS.

Our study has several limitations. First, the large number of heterogeneous trials creates the potential for several confounders. The general interpretability of the results would be challenged without adequate adjustment for patient characteristics that arise from differences in predefined eligibility criteria for clinical trial enrollment. Specifically, our analysis did not distinguish between platinum-sensitive and platinum-resistant ovarian cancer patients. Because platinum sensitivity influences OS greatly, no consideration of that status may have confounded our analysis. There were too few cases in either category to justify separate analyses that, as a consequence of the small sample size, would have been poorly powered, however the ratio of PPS/OS looked similar regardless of platinum sensitivity. No imbalance was detected overall or between older and recent studies including studies that had a pooled analysis for patients sensitive or resistant to platinum-based chemotherapy. Oza et al. found a correlation between PFS and OS in trials of first-line cytotoxic chemotherapy, however it remains unclear whether this holds true for trials incorporating molecular targeted agents [34]. While our study looked at second/third-line treatments, we did not distinguish between cytotoxic chemotherapy and molecular targeted agents, and thus our results may have been biased because both types of patients were pooled. Secondly, in characterizing the relationship between OS and endpoints based on tumor assessment, individual patient data are considered ideal; however, this study analysis was based on abstracted data. This was the case in part because we did not have full access to individual patient data. Nonetheless, the use of abstracted data from available publications enables independent replication of the analysis. Thirdly, disease progression is sometimes vulnerable to measurement error and the influence of bias in patient assessment. Also, there may have been inconsistencies in the quality of endpoint measurements based on tumor assessment (PFS or TTP) across centers and trials. Lastly, we regarded both PFS and TTP are the same variable in our study. In fact, PFS is defined as the time from randomization to objective tumor progression or death. TTP meanwhile censors death and is instead defined only as the time from randomization to objective tumor progression. If 
death does not occur during treatment, there is no difference between these two endpoints. In ovarian cancer, disease progression is seldom preceded by death. Thus, we determined PFS and TTP as interchangeable.

Our study demonstrated that the correlation between OS and PPS is strong while the correlation between OS and PFS is modest, and that this trend has become pronounced over time. Any correlation between PFS and OS in platinum-sensitive epithelial ovarian cancer remains inconclusive, and in platinum-resistant epithelial ovarian cancer, PFS is not a recommended endpoint over improved OS or QOL [34]. The OS of patients with advanced or recurrent epithelial ovarian cancer receiving secondor third-line therapy is not as prolonged as it is in other diseases because only a small proportion of patients undergo subsequent therapy after progression. Due to the limited number of post-trial therapies in second/third-line advanced or recurrent epithelial ovarian cancer, fewer confounding variables are expected in comparison to first-line trials, although newly invented treatment (e.g. PARP inhibitors) may be tested as a clinical trial. Given that the correlation between OS and PFS has become weaker in recent years, this study supports the selection of OS as an appropriate primary endpoint in clinical trials for second/third-line patients with advanced or recurrent epithelial ovarian cancer, however PFS is always reasonable surrogate of OS and our result supported its association.

\section{Supplementary Material}

Table S1. http://www.jcancer.org/v09p0872s1.xlsx Table S2. http://www.jcancer.org/v09p0872s2.pdf

\section{Competing Interests}

The authors have declared that no competing interest exists.

\section{References}

1. Jemal A, Bray F, Center MM, et al. Global cancer statistics. CA Cancer J Clin. 2011; 61: 69-90.

2. American Cancer Society. Cancer Facts \& Figures 2015. Atlanta: American Cancer Society; 2015.

3. Colombo N, Peiretti M, Parma G, et al. Newly diagnosed and relapsed epithelial ovarian carcinoma: ESMO Clinical Practice Guidelines for diagnosis, treatment and follow-up. Ann Oncol. 2010; 21 (suppl 5): v23-30.

4. Kim A, Ueda Y, Naka T, et al. Therapeutic strategies in epithelial ovarian cancer. J Exp Clin Cancer Res. 2012; 31: 14.

5. Heintz AP, Odicino F, Maisonneuve P, et al. Carcinoma of the ovary. FIGO 26th Annual Report on the Results of Treatment in Gynecological Cancer. Int J Gynaecol Obstet. 2006; 95 (Suppl 1): S161-S192.

6. Ozols RF. Systemic therapy for ovarian cancer: current status and new treatments. Semin Oncol. 2006; 33 (2 Suppl 6): S3-11.

7. Markman M. The waning relationship between progression-free survival and overall survival in randomized cancer therapy trials. Am J Hematol Oncol. 2014; 10: 30-32.

8. Shimokwa M, Ohki M, Kaku T. Correlation of progression-free and post-progression survival with overall survival in phase III trials of first-line chemotherapy for advanced epithelial ovarian cancer. Eur J Gynaecol Oncol. $2015 ; 36: 370-5$.
9. Saad ED, Katz A, Buyse M. Overall survival and post-progression survival in advanced breast cancer: a review of recent randomized clinical trials. J Clin Oncol. 2010; 28: 1958-62.

10. Hayashi H, Okamoto I, Morita S, et al. Postprogression survival for first-line chemotherapy of patients with advanced non-small-cell lung cancer. Ann Oncol. 2012; 23: 1537-41.

11. Gordon AN, Fleagle JT, Guthrie D, et al. Recurrent epithelial ovarian carcinoma: a randomized phase III study of pegylated liposomal doxorubicin versus topotecan. J Clin Oncol. 2001; 19: 3312-22.

12. Muggia F, Hamilton A. Phase III data on Caelyx in ovarian cancer. Eur J Cancer. 2001; 37 (Suppl 9): S15-8.

13. Cantu MG, Buda A, Parma G, et al. Randomized controlled trial of single-agent paclitaxel versus cyclophosphamide, doxorubicin, and cisplatin in patients with recurrent ovarian cancer who responded to first-line platinum-based regimens. J Clin Oncol. 2002; 20: 1232-7.

14. Parmar MK, Ledermann JA, Colombo N, et al. Paclitaxel plus platinum-based chemotherapy versus conventional platinum-based chemotherapy in women with relapsed ovarian cancer: the ICON4/AGO-OVAR-2.2 trial. Lancet. 2003; 361: 2099-106.

15. Omura GA, Brady MF, Look KY, et al. Phase III trial of paclitaxel at two dose levels, the higher dose accompanied by filgrastim at two dose levels in platinum-pretreated epithelial ovarian cancer: an intergroup study. J Clin Oncol. 2003; 21: 2843-8.

16. Buda A, Floriani I, Rossi R, et al. Randomised controlled trial comparing single agent paclitaxel vs epidoxorubicin plus paclitaxel in patients with advanced ovarian cancer in early progression after platinum-based chemotherapy: an Italian Collaborative Study from the Mario Negri Institute, Milan, G.O.N.O. (Gruppo Oncologico Nord Ovest) group and I.O.R. (Istituto Oncologico Romagnolo) group. Br J Cancer. 2004; 90: 2112-7.

17. ten Bokkel Huinink W, Lane SR, Ross GA, et al. Long-term survival in a phase III, randomised study of topotecan versus paclitaxel in advanced epithelial ovarian carcinoma. Ann Oncol. 2004; 15: 100-3.

18. Pfisterer J, Plante M, Vergote I, et al. Gemcitabine plus carboplatin compared with carboplatin in patients with platinum-sensitive recurrent ovarian cancer: an intergroup trial of the AGO-OVAR, the NCIC CTG, and the EORTC GCG. J Clin Oncol. 2006; 24: 4699-707.

19. Hirte $\mathrm{H}$, Vergote IB, Jeffrey JR, et al. A phase III randomized trial of BAY 12-9566 (tanomastat) as maintenance therapy in patients with advanced ovarian cancer responsive to primary surgery and paclitaxel/platinum containing chemotherapy: a National Cancer Institute of Canada Clinical Trials Group Study. Gynecol Oncol. 2006; 102: 300-8.

20. Mutch DG, Orlando M, Goss T, et al. Randomized phase III trial of gemcitabine compared with pegylated liposomal doxorubicin in patients with platinum-resistant ovarian cancer. J Clin Oncol. 2007; 25: 2811-8.

21. Sehouli J, Stengel D, Oskay-Oezcelik G, et al. Nonplatinum topotecan combinations versus topotecan alone for recurrent ovarian cancer: results of a phase III study of the North-Eastern German Society of Gynecological Oncology Ovarian Cancer Study Group. J Clin Oncol. 2008; 26: 3176-82.

22. Ferrandina G, Ludovisi $M$, Lorusso D, et al. Phase III trial of gemcitabine compared with pegylated liposomal doxorubicin in progressive or recurrent ovarian cancer. J Clin Oncol. 2008; 26: 890-6.

23. Markman M, Liu PY, Moon J, et al. Impact on survival of 12 versus 3 monthly cycles of paclitaxel $(175 \mathrm{mg} / \mathrm{m} 2)$ administered to patients with advanced ovarian cancer who attained a complete response to primary platinum-paclitaxel: follow-up of a Southwest Oncology Group and Gynecologic Oncology Group phase 3 trial. Gynecol Oncol. 2009; 114: 195-8.

24. Vergote I, Finkler N, del Campo J, et al. Phase 3 randomised study of canfosfamide (Telcyta, TLK286) versus pegylated liposomal doxorubicin or topotecan as third-line therapy in patients with platinum-refractory or -resistant ovarian cancer. Eur J Cancer. 2009; 45: 2324-32.

25. Meier W, du Bois A, Reuss A, et al. Topotecan versus treosulfan, an alkylating agent, in patients with epithelial ovarian cancer and relapse within 12 months following 1st-line platinum/paclitaxel chemotherapy. A prospectively randomized phase III trial by the Arbeitsgemeinschaft Gynaekologische Onkologie Ovarian Cancer Study Group (AGO-OVAR). Gynecol Oncol. 2009; 114: 199-205.

26. Hurteau JA, Brady MF, Darcy KM, et al. Randomized phase III trial of tamoxifen versus thalidomide in women with biochemical-recurrent-only epithelial ovarian, fallopian tube or primary peritoneal carcinoma after a complete response to first-line platinum/taxane chemotherapy with an evaluation of serum vascular endothelial growth factor (VEGF): A Gynecologic Oncology Group Study. Gynecol Oncol. 2010; 119: 444-50.

27. Markman M, Moon J, Wilczynski S, et al. Single agent carboplatin versus carboplatin plus pegylated liposomal doxorubicin in recurrent ovarian cancer: final survival results of a SWOG (S0200) phase 3 randomized trial. Gynecol Oncol. 2010; 116: 323-5.

28. Monk BJ, Herzog TJ, Kaye SB, et al. Trabectedin plus pegylated liposomal doxorubicin (PLD) versus PLD in recurrent ovarian cancer: overall survival analysis. Eur J Cancer. 2012; 48: 2361-8.

29. Colombo N, Kutarska E, Dimopoulos M, et al. Randomized, open-label, phase III study comparing patupilone (EPO906) with pegylated liposomal doxorubicin in platinum-refractory or -resistant patients with recurrent epithelial ovarian, primary fallopian tube, or primary peritoneal cancer. J Clin Oncol. 2012; 30: 3841-7 
30. Wagner U, Marth C, Largillier R, et al. Final overall survival results of phase III GCIG CALYPSO trial of pegylated liposomal doxorubicin and carboplatin vs paclitaxel and carboplatin in platinum-sensitive ovarian cancer patients. $\mathrm{Br}$ J Cancer. 2012; 107: 588-91.

31. Pujade-Lauraine E, Hilpert F, Weber B, et al. Bevacizumab Combined With Chemotherapy for Platinum-Resistant Recurrent Ovarian Cancer: The AURELIA open-label randomized phase III trial. J Clin Oncol. 2014; 32: 1302-8.

32. Fotopoulou C, Vergote I, Mainwaring P, et al. Weekly AUC2 carboplatin in acquired platinum-resistant ovarian cancer with or without oral phenoxodiol, a sensitizer of platinum cytotoxicity: the phase III OVATURE multicenter randomized study. Ann Oncol. 2014; 25: 160-5.

33. ten Bokkel Huinink W, Gore M, Carmichael J, et al. Topotecan versus paclitaxel for the treatment of recurrent epithelial ovarian cancer. J Clin Oncol. 1997; 15: 2183-93.

34. Oza AM, Castonguay V, Tsoref D, et al. Progression-free survival in advanced ovarian cancer: a Canadian review and expert panel perspective. Curr Oncol. 2011; 18 (Suppl 2): S20-7.

35. Broglio KR, Berry DA. Detecting an overall survival benefit that is derived from progression-free survival. J Natl Cancer Inst. 2009; 101: 1642-9.

36. Reig M, Rimola J, Torres F, et al. Postprogression survival of patients with advanced hepatocellular carcinoma: rationale for second-line trial design. Hepatology. 2013; 58: 2023-31.

37. Paoletti X, Oba K, Bang YJ, et al. Progression-free survival as a surrogate for overall survival in advanced/recurrent gastric cancer trials: a meta-analysis. J Natl Cancer Inst. 2013; 105: 1667-70.

38. Buyse M, Burzykowski T, Carroll K, et al. Progression-free survival is a surrogate for survival in advanced colorectal cancer. J Clin Oncol. 2007; 25: $5218-24$

39. Petrelli F, Barni S. Correlation of progression-free and post-progression survival with overall survival in advanced colorectal cancer. Ann Oncol. 2013; 24: $186-92$. 